\title{
Active Control of Scattered Acoustic Fields: Cancellation, Reproduction and Cloaking
}

\author{
Jordan Cheer \\ j.cheer@soton.ac.uk \\ Institute of Sound and Vibration Research, University of Southampton, \\ University Road, Southampton SO17 1BJ, UK
}

August 112016

\begin{abstract}
The active control of sound fields has been widely applied in both active noise control and sound field reproduction, however, relatively few studies have focused on active acoustic cloaking. In order to build upon the knowledge and understanding in the areas of active noise control and sound field reproduction, this paper investigates their physical limitations and compares them to the active cloaking problem when the three strategies are employed in the presence of an acoustic scatterer. The three sound field control strategies have been formulated within a consistent framework, and this has enabled insight into the physical control mechanisms. Two different three-dimensional scattering problems have then been simulated and used to investigate the performance limitations of the three strategies. The influence of the number of control sources and their proximity to the scattering object have been investigated, and it has been shown that the requirements for active cloaking differ from those for active noise control and sound field reproduction. Specifically, it has been shown that there is a clear distinction between controlling the internal and external sound fields in the three cases.
\end{abstract}

\section{Introduction}

Sound field control is the method of actively manipulating an acoustic field using control, or secondary sources. This technology encompasses active noise control [1], sound field reproduction [2] and active acoustic cloaking, 
or the control of scattered sound fields [3]. In the context of active noise control, the control sources are used to cancel, or attenuate, the sound field produced by a primary acoustic disturbance. This technology has been exploited in a variety of applications where passive control treatments are not able to achieve sufficient performance within the defined size and weight constraints. For example, active noise control has been used in the automotive environment to control low frequency engine and road noise using the car audio loudspeakers $[4,5,6,7]$. In the context of sound field reproduction, the control sources are instead used to generate a desired sound field. For example, at a fundamental level, an array of control sources could be used to reproduce a plane wave [8], however, this can be extended to achieve the generation of multiple listening zones in a single acoustic space $[9,10,11,12]$, and the generation of fully immersive artificial acoustic environments that accurately synthesise a real acoustic experience $[13,14]$. Finally, in active acoustic cloaking the control sources are used to cancel the sound field scattered by an object, with the aim of making that object acoustically 'invisible'. Different methods of active acoustic cloaking have been investigated through both simulation $[15,16]$ and experimentation $[3,17,18]$.

Although the three sound field control technologies have different objectives - cancellation, reproduction or cloaking - it is necessary in each case to solve a similar control problem. The physical limitations on active noise control and sound field reproduction are relatively well understood [1, 2], however, there have been fewer exhaustive studies into the limitations of active acoustic cloaking. Therefore, this paper investigates the physical limitations on the three different sound field control technologies within a consistent framework so that the developed understanding of the cancellation and reproduction problems can be used to provide insight into the relatively underdeveloped problem of active acoustic cloaking.

The physical limitations of active noise control and sound field reproduction have been extensively investigated [1,2] and, in general, these control strategies are limited to relatively low frequencies. This is because, in many applications, the number of control sources required to achieve either accurate secondary field generation in active control or accurate sound field reproduction increases as the wavelength of sound becomes smaller. Despite its potential applications, there have been fewer studies on the limitations of active acoustic cloaking. For example, Bobrovnitskii has proposed an active acoustic cloaking method based on using structural sensors and actuators on an elastic scattering body to detect and control the scattered sound field respectively[19, 16]. Although this method is shown to theoretically achieve perfect cancellation [20] and avoids the need for sources and sensors located remotely from the scatterer [16], it has not been practically demonstrated. In [16] it has been shown for the case of an infinite cylinder how increasing the number of 
structural control sources increases both the level and bandwidth of control. An alternative method of active acoustic cloaking has been proposed and practically demonstrated by Friot et al $[3,17]$ which uses loudspeakers to control the scattered field and a dual layer of microphones to detect the scattered field. However, in this case a comprehensive study into the effects of the number and distribution of the control sources has not been presented. Therefore, as stated above, this paper aims to extend this previous work on active acoustic cloaking by presenting an investigation into the physical limits of active cloaking, with a particular focus on how the number and geometrical distribution of control sources influences the control performance.

In order to place active acoustic cloaking in context, Section II first reviews the formulations of both active noise control and sound field reproduction, before deriving the active acoustic cloaking formulation within the same framework. This formulation of active acoustic cloaking facilitates a direct comparison to the other sound field control technologies that has not previously been explicitly discussed and provides a new insight into the physical mechanisms of control. In Section III a model of a three dimensional acoustic scattering problem is presented and in Section IV this model is used to investigate the physical limitations on the three sound field control strategies. In particular, the influence of the control frequency, the number of secondary sources and the distance between the scattering object and the control sources are investigated. In Section V a more complex scattering problem is considered and finally, in Section VI, conclusions are drawn.

\section{Formulations of Sound Field Control}

The three sound field control problems considered in this paper have been approached from a number of different perspectives in the literature and, therefore, there has not been a consistent framework within which the three problems can be directly compared in order to provide insight into the physical limitations of the three strategies. Therefore, in the following sections the three sound field control problems are formulated in terms of the acoustic pressures, source strengths and associated acoustic transfer responses.

\section{A Active Noise Control}

In active noise control applications, secondary or control sources are used to cancel the sound field produced by an unwanted primary source. For example, in the automotive application this could be the sound field produced in the car cabin due to the interaction between the road and tyre. Considering the multichannel control problem where $M$ control sources are used to minimise the pressure at $L$ error sensors, the vector of 
complex error signals at a single frequency can be expressed as

$$
\mathbf{p}=\mathbf{p}_{p}+\mathbf{Z q}
$$

where $\mathbf{p}_{p}$ is the vector of the pressures due to the primary source at the $L$ error sensors, $\mathbf{Z}$ is the $L \times M$ matrix of complex transfer responses between the $M$ control sources and $L$ error sensors and $\mathbf{q}$ is the vector of $M$ control source strengths. The general aim of an active noise control system is to minimise the vector of error signals and this can be achieved most straightforwardly by minimising the cost function given by the sum of the squared error signals. However, it is often necessary to also include a term in the cost function that is related to the power required by the controller, and this can be achieved by including a term in the cost function that is proportional to the control effort, or the sum of the modulus squared control source strengths, $\mathbf{q}^{H} \mathbf{q}$. The cost function in this case is

$$
J_{A N C}=\mathbf{p}^{H} \mathbf{p}+\beta \mathbf{q}^{H} \mathbf{q}
$$

where superscript $H$ denotes the Hermitian, complex conjugate transpose and $\beta$ is the positive real effortweighting parameter. Substituting Eq. (1) into Eq. (2) leads to the Hermitian quadratic function

$$
J_{A N C}=\mathbf{q}^{H}\left[\mathbf{Z}^{H} \mathbf{Z}+\beta \mathbf{I}\right] \mathbf{q}+\mathbf{p}_{p}^{H} \mathbf{Z} \mathbf{q}+\mathbf{q}^{H} \mathbf{Z}^{H} \mathbf{p}_{p}+\mathbf{p}_{p}^{H} \mathbf{p}_{p}
$$

The optimal set of control source strengths that minimises $J_{A N C}$ can then be calculated by setting the differential of Eq. (3) with respect to the real and imaginary parts of $\mathbf{q}$ to zero [1]. The differential of Eq. (3) with respect to the real and imaginary parts of $\mathbf{q}$ is

$$
\frac{\partial J_{A N C}}{\partial \mathbf{q}}=2\left[\mathbf{Z}^{H} \mathbf{Z}+\beta \mathbf{I}\right] \mathbf{q}+2 \mathbf{Z}^{H} \mathbf{p}_{p}
$$

and equating this to zero and rearranging gives the optimal vector of control source strengths that minimises the cost function given by Eq. (2) as

$$
\mathbf{q}_{A N C}=-\left[\mathbf{Z}^{H} \mathbf{Z}+\beta \mathbf{I}\right]^{-1} \mathbf{Z}^{H} \mathbf{p}_{p}
$$


The minimum value of the cost function, $J_{A N C}$, can then be found by setting $\beta=0$, to remove the constraint on the control effort, and substituting Eq. (5) into Eq. (3), which gives

$$
\min \left\{J_{A N C}\right\}=\mathbf{p}_{p}^{H}\left[\mathbf{I}-\mathbf{Z}\left[\mathbf{Z}^{H} \mathbf{Z}\right]^{-1} \mathbf{Z}^{H}\right] \mathbf{p}_{p}
$$

\section{B Sound Field Reproduction}

In sound field reproduction, rather than interacting with the sound field produced by a primary source, the control sources are driven in order to produce a desired, or target sound field. In this case the control source optimisation problem can be formulated by defining the sound field reproduction error signal as the difference between the target sound field, $\mathbf{p}_{T}$, and the sound field produced by the control sources, $\mathbf{Z} \mathbf{q}$, that is,

$$
\mathbf{e}_{S F R}=\mathbf{Z q}-\mathbf{p}_{T}
$$

The cost function to be minimised can then be expressed as

$$
J_{S F R}=\mathbf{e}_{S F R}^{H} \mathbf{e}_{S F R}+\beta \mathbf{q}^{H} \mathbf{q}
$$

where, once again, a term proportional to the control effort has been included in addition to the sum of the squared reproduction errors. By substituting Eq. (7) into the cost function then gives

$$
J_{S F R}=\mathbf{q}^{H}\left[\mathbf{Z}^{H} \mathbf{Z}+\beta \mathbf{I}\right] \mathbf{q}-\mathbf{p}_{T}^{H} \mathbf{Z} \mathbf{q}-\mathbf{q}^{H} \mathbf{Z}^{H} \mathbf{p}_{T}+\mathbf{p}_{T}^{H} \mathbf{p}_{T}
$$

As in the previous section, the optimal vector of control source strengths can then be derived by setting the differential of Eq. (9) with respect to the real and imaginary parts of $\mathbf{q}$ to zero. This gives the optimal vector of control source strengths for the sound field reproduction problem as

$$
\mathbf{q}_{S F R}=\left[\mathbf{Z}^{H} \mathbf{Z}+\beta \mathbf{I}\right]^{-1} \mathbf{Z}^{H} \mathbf{p}_{T}
$$

In comparison to the optimal control source strengths for the active noise control application given by Eq. (5), it can be seen that in the sound field reproduction solution the disturbance vector, $\mathbf{p}_{p}$, is replaced with the desired field vector, $\mathbf{p}_{T}$, and the negative sign is removed. This is because in the active noise control application the control sources attempt to achieve cancellation by reproducing the primary sound field but 
out-of-phase, conversely, in the sound field reproduction problem the control sources attempt to reproduce the desired field without any phase inversion. This difference can also be observed in terms of the minimum of the cost function, $J_{S F R}$, which is obtained by setting $\beta=0$ and substituting Eq. (10) into Eq. (9) to give

$$
\min \left\{J_{S F R}\right\}=\mathbf{p}_{T}^{H}\left[\mathbf{I}-\mathbf{Z}\left[\mathbf{Z}^{H} \mathbf{Z}\right]^{-1} \mathbf{Z}^{H}\right] \mathbf{p}_{T}
$$

From this it can be seen that the minimum of this cost function depends on the target pressure vector, $\mathbf{p}_{T}$, rather than the primary pressure vector that determined the minimum of the active noise control cost function given by Eq. (6).

\section{Active Acoustic Cloaking}

In addition to the standard sound field control problems, active acoustic cloaking has also recently been of interest in a number of applications. In this configuration, the aim of the sound field control system is to minimise the influence of a scattering object on the sound field produced by a primary source and, thus, cloak the scattering object. As such, the control sources must be driven to minimise the scattered sound field, whilst leaving the sound field due to the primary source unaltered. In this formulation, the sound field at the error sensors can be expressed as given by Eq. (1) for the active noise control application, since the sound field is due to both the primary and secondary sources in this scenario. However, in the case of acoustic cloaking the vector of error signals that should be minimised is given by the difference between the total pressure field after control, $\mathbf{p}$, and the target pressure field, $\mathbf{p}_{T}$, which is

$$
\begin{aligned}
\mathbf{e}_{c} & =\mathbf{p}-\mathbf{p}_{T}, \\
& =\mathbf{p}_{p}+\mathbf{Z} \mathbf{q}-\mathbf{p}_{T} .
\end{aligned}
$$

In the case of acoustic cloaking, the sound field that is targeted after control, $\mathbf{p}_{T}$, is generally the sound field produced by the primary source in the absence of the scattering object. This may, for example, be the sound field produced by the primary source under free field conditions. It can be seen from the error vector given by Eq. (12) that, compared to the sound field reproduction error vector given by Eq. (7), there is also a component due to the primary source.

Following the formulations of the active noise control and sound field reproduction problems, the cost 
function for the active acoustic cloaking problem can be defined as

$$
J_{c}=\mathbf{e}_{c}^{H} \mathbf{e}_{c}+\beta \mathbf{q}^{H} \mathbf{q}
$$

Substituting Eq. (12) for $\mathbf{e}_{c}$ into the cost function then leads to

$$
\begin{aligned}
J_{c} & =\left(\mathbf{p}_{p}^{H}+\mathbf{q}^{H} \mathbf{Z}^{H}-\mathbf{p}_{T}^{H}\right)\left(\mathbf{p}+\mathbf{Z} \mathbf{q}-\mathbf{p}_{T}\right)+\beta \mathbf{q}^{H} \mathbf{q} \\
& =\mathbf{q}^{H}\left[\mathbf{Z}^{H} \mathbf{Z}+\beta \mathbf{I}\right] \mathbf{q}-\mathbf{p}_{T}^{H} \mathbf{Z} \mathbf{q}-\mathbf{q}^{H} \mathbf{Z}^{H} \mathbf{p}_{T}+\mathbf{p}_{p}^{H} \mathbf{Z} \mathbf{q}+\mathbf{q}^{H} \mathbf{Z}^{H} \mathbf{p}_{p}-\mathbf{p}_{T}^{H} \mathbf{p}_{p}-\mathbf{p}_{p}^{H} \mathbf{p}_{T}+\mathbf{p}_{T}^{H} \mathbf{p}_{T}+\mathbf{p}_{p}^{H} \mathbf{p}_{p}
\end{aligned}
$$

which can be written in the standard Hermitian quadratic form as

$$
J_{c}=\mathbf{q}^{H}\left[\mathbf{Z}^{H} \mathbf{Z}+\beta \mathbf{I}\right] \mathbf{q}+\left(\mathbf{p}_{p}-\mathbf{p}_{T}\right)^{H} \mathbf{Z} \mathbf{q}+\mathbf{q}^{H} \mathbf{Z}^{H}\left(\mathbf{p}_{p}-\mathbf{p}_{T}\right)+\left(\mathbf{p}_{p}-\mathbf{p}_{T}\right)^{H}\left(\mathbf{p}_{p}-\mathbf{p}_{T}\right) .
$$

Differentiating this cost function with respect to the real and imaginary parts of $\mathbf{q}$ gives

$$
\frac{\partial J_{c}}{\partial \mathbf{q}}=2\left[\mathbf{Z}^{H} \mathbf{Z}+\beta \mathbf{I}\right] \mathbf{q}+2 \mathbf{Z}^{H}\left(\mathbf{p}_{p}-\mathbf{p}_{T}\right)
$$

and equating this to zero and rearranging gives the optimal vector of control source strengths for the active acoustic cloaking application as

$$
\mathbf{q}_{c}=-\left[\mathbf{Z}^{H} \mathbf{Z}+\beta \mathbf{I}\right]^{-1} \mathbf{Z}^{H}\left(\mathbf{p}_{p}-\mathbf{p}_{T}\right) .
$$

Comparing this solution for the vector of optimal control source strengths to the solutions for the active noise control and sound reproduction problems, given by Eqs. (5) and (10) respectively, it can be seen that the active acoustic cloaking solution is a linear combination of the solutions for the two standard sound field control methods. This suggests that the operation of the active acoustic cloaking system is to cancel the primary sound field produced in the presence of the scattering object, and then reproduce the primary sound field that would occur in the absence of the scattering object. However, physically, the active acoustic cloaking system is only required to cancel the scattered sound field. This can be demonstrated by using the fact that the sound field produced by the primary source in the presence of a scattering object can be 
expressed as the linear superposition of the free field and the scattered primary pressures

$$
\mathbf{p}_{p}=\mathbf{p}_{p_{F}}+\mathbf{p}_{p_{S}}
$$

where $\mathbf{p}_{p_{F}}$ is the primary sound field in the absence of the scattering object, and $\mathbf{p}_{p_{S}}$ is the primary sound field due to the scattering. In the case of acoustic cloaking, the target sound field is set equal to the primary sound field without scattering, that is $\mathbf{p}_{T}=\mathbf{p}_{p_{F}}$. Making this assumption and substituting Eq. (19) into Eq. (12) gives the error vector as

$$
\begin{aligned}
\mathbf{e}_{c} & =\mathbf{p}_{p_{F}}+\mathbf{p}_{p_{S}}+\mathbf{Z q}-\mathbf{p}_{p_{F}}, \\
& =\mathbf{p}_{p_{S}}+\mathbf{Z q} .
\end{aligned}
$$

The cloaking cost function can then equivalently be written as

$$
J_{c}=\mathbf{q}^{H}\left[\mathbf{Z}^{H} \mathbf{Z}+\beta \mathbf{I}\right] \mathbf{q}+\mathbf{p}_{p_{s}}^{H} \mathbf{Z} \mathbf{q}+\mathbf{q}^{H} \mathbf{Z}^{H} \mathbf{p}_{p_{s}}+\mathbf{p}_{p_{s}}^{H} \mathbf{p}_{p_{s}}
$$

and the optimal vector of control source strengths can be expressed as

$$
\mathbf{q}_{c}=-\left[\mathbf{Z}^{H} \mathbf{Z}+\beta \mathbf{I}\right]^{-1} \mathbf{Z}^{H} \mathbf{p}_{p_{s}}
$$

The minimum cost function in this case is then given by setting $\beta=0$ and substituting Eq. (22) into Eq. (21), which gives

$$
\min \left\{J_{c}\right\}=\mathbf{p}_{p_{s}}^{H}\left[\mathbf{I}-\mathbf{Z}\left[\mathbf{Z}^{H} \mathbf{Z}\right]^{-1} \mathbf{Z}^{H}\right] \mathbf{p}_{p_{s}} .
$$

From Eq. (22) it can be seen that the active acoustic cloaking system only cancels the primary sound field due to scattering, and as a result, the minimum of the cost function given by Eq. (23) is only dependent on the scattered primary field.

It is interesting to compare the result given by Eq. (23) to the corresponding results for the active noise control and sound field reproduction strategies. For the case when the sound field due to the primary source is given by the superposition of the free field and scattered primary pressures, as in Eq. (19), the minimum of the active noise control cost function, $J_{A N C}$, given by Eq. (6) will depend on both the free field and scattered primary pressures. However, when the target pressure in the sound field reproduction strategy is 
defined as the free field primary pressure, $\mathbf{p}_{T}=\mathbf{p}_{p_{F}}$, then the minimum of the cost function $J_{S F R}$ given by Eq. (11) will only depend on the free field primary pressure. The influence that these observations have on the physical limitations of the three sound field control strategies will be explored in the following sections.

\section{A Simple Acoustical Scattering Problem}

The main aim of this paper is to provide physical insight into the sound field control problem of acoustic cloaking by building on the more developed understanding of both active noise control and sound field reproduction. In order to study the active acoustic cloaking problem it is necessary to consider an acoustical scenario in which there is scattering from an object, as the focus in this case is fundamentally on controlling the scattered sound field. The problem of controlling the sound field in the presence of a scattering object is, however, not an artificial construct in the context of active noise control and sound field reproduction. Both of these technologies are often used to control the sound field around a listener's head, which acts as a scattering object, and therefore considering the application of these control problems to an acoustical scattering problem has practical relevance.

In order to investigate the physical limits on the three sound field control strategies, a three-dimensional acoustic scattering problem has been modelled using the finite element method. The primary sound field is generated by a plane wave and the scattering object has been defined as a rigid sphere with a radius of $a=0.2 \mathrm{~m}$, as shown in Fig. 1 . The pressure has been evaluated over a cubic volume of $1 \mathrm{~m}^{3}$ and the dots in Fig. 1 show a single plane of this three-dimensional pressure evaluation grid. The maximum separation between the pressure evaluation points in this three-dimensional grid has been set to give a minimum of 10 observation points per wavelength at the maximum frequency of interest and, therefore, limitations due to observability are avoided. A total of 66812 pressure evaluation positions have been employed and these are used in the following sections as the error sensor pressures. In order to limit the modelling problem to a finite size, a Perfectly Matched Layer (PML) has been used to truncate the acoustic domain [21]. Finally, the secondary sources used in the three control strategies have been defined as monopoles, and these are shown for one secondary source geometry as the crosses surrounding the rigid sphere in Fig. 1. Although the problem of scattering from a rigid sphere could be modelled analytically using spherical harmonics [22, 23], a finite element model has been employed to allow future extensions to more complex scattering problems, as considered in Section V.

The accuracy of the finite element model has been validated through a convergence study and the thickness 


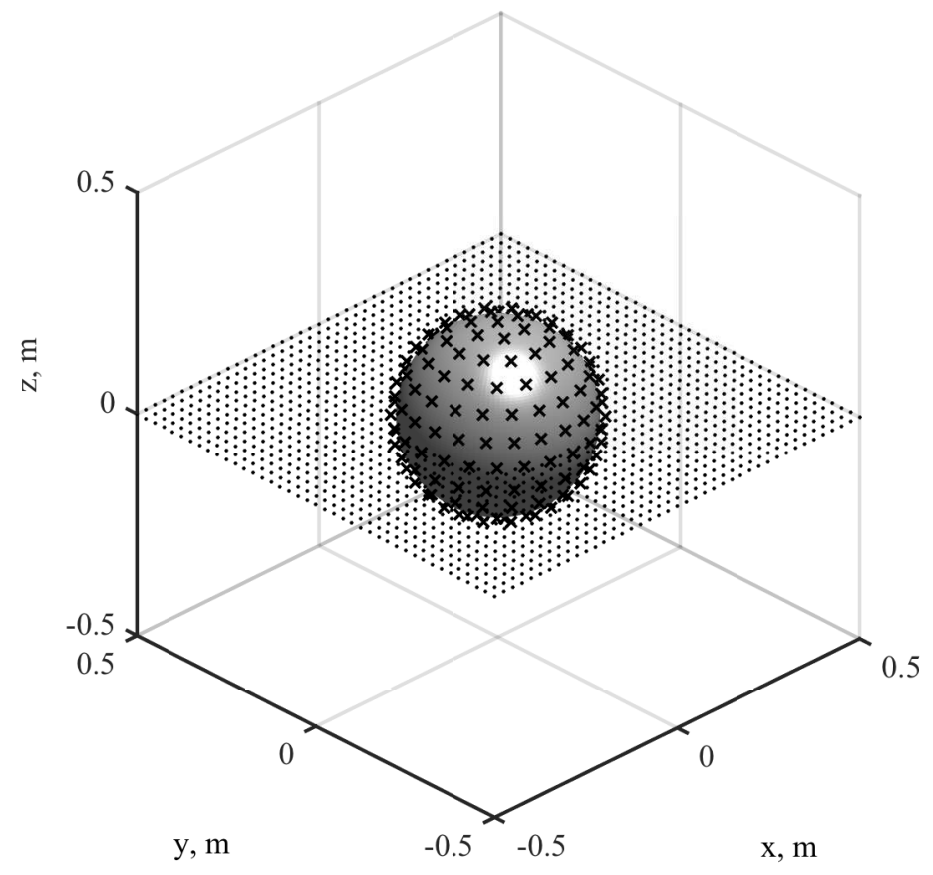

Figure 1: The geometry of the spherical scatterer, the control sources for the interior control geometry (crosses), and a single plane of the full three-dimensional grid of error sensors (dots). 


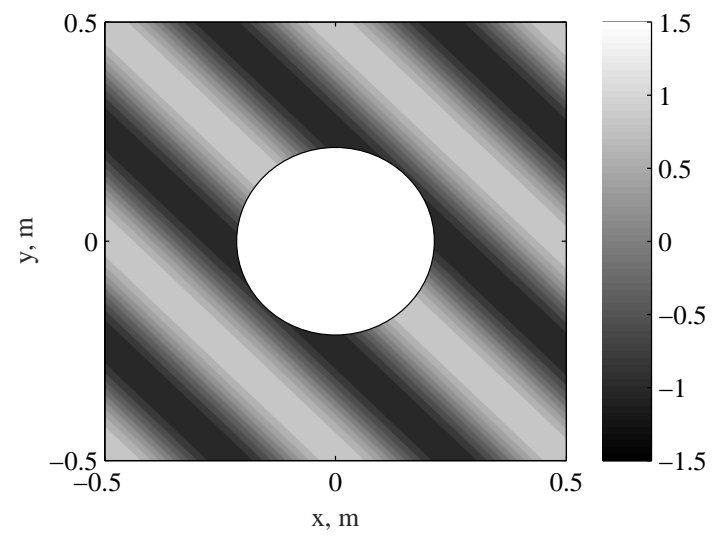

(a)

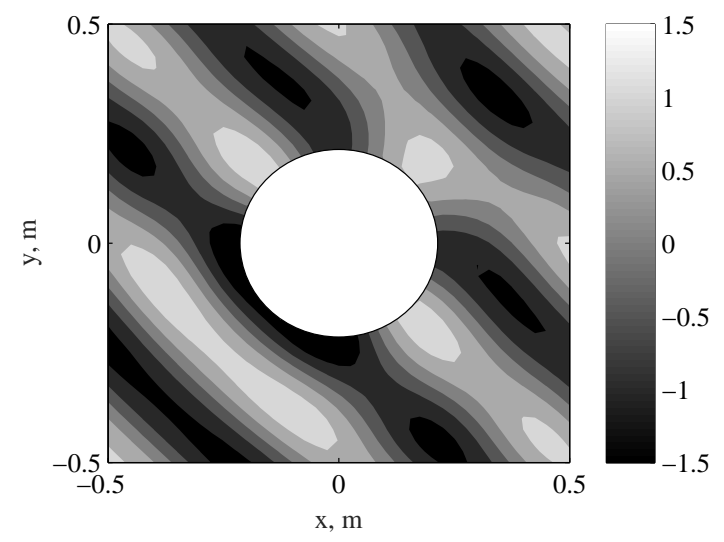

(b)

Figure 2: A two-dimensional slice through the three-dimensional primary sound field at $1 \mathrm{kHz}$ (a) without scattering and (b) with scattering.

of the PML has been defined at each frequency to ensure that outgoing waves are sufficiently attenuated and reflections back into the domain of interest do not occur. An example of the primary sound field with and without the scattering object is shown in Fig. 2 for an excitation frequency of $1 \mathrm{kHz}$. From Fig. 2a it can be seen that the primary sound field without scattering, that is the free field primary pressure $\mathbf{p}_{p_{F}}$, is a plane wave.

\section{Physical Limitations on Sound Field Control}

As discussed in the introduction, the physical limitations on both active noise control and sound field reproduction have been extensively investigated $[1,2]$. In addition to the limitation on the frequency range over which effective sound field control can be achieved in these two cases, there are also fundamental differences between attempting to control the sound field within an interior region enclosed by the array of control sources and an exterior region outside of the array of control sources. Therefore, in following sections, in addition to considering the effect of frequency on the control performance, the exterior and interior control problems will also be explored.

\section{A The Exterior Control Problem}

In the first instance, the limitations on the three sound field control strategies have been investigated for a single layer of 180 control sources equally distributed around the rigid spherical scatterer at a small distance 


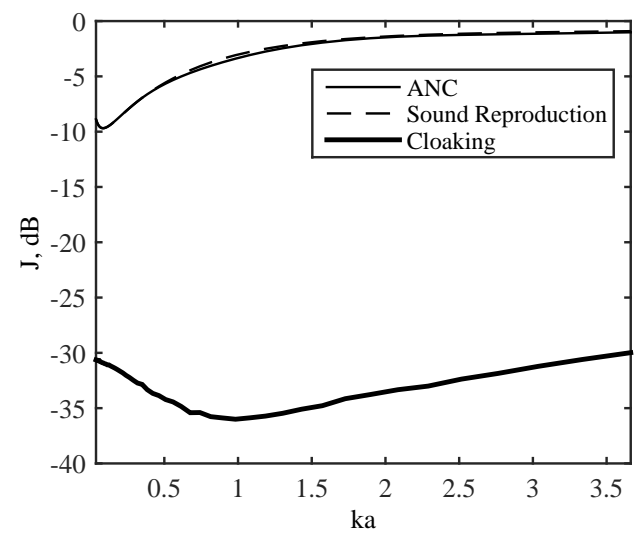

(a)

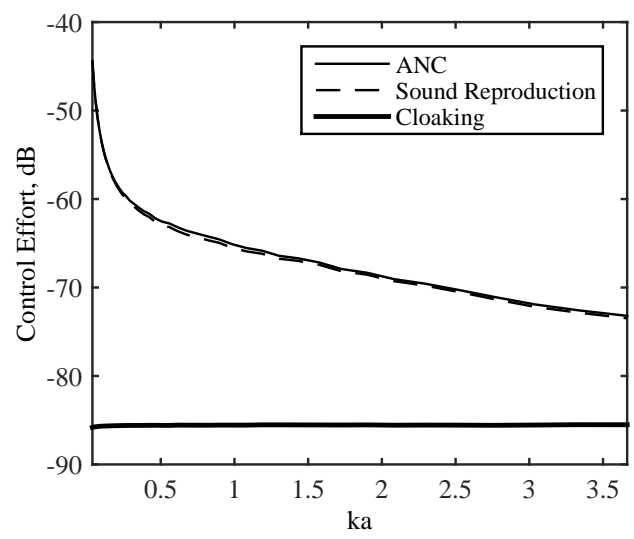

(b)

Figure 3: The attenuation in the cost functions (a) and the corresponding control efforts (b) for the active noise control, sound field reproduction and active acoustic cloaking control schemes for a single layer array of 180 control sources uniformly distributed over the scattering sphere controlling the exterior sound field.

of $a / 20$ from the surface of the sphere, as shown in Fig. 1. As described in Section III, the error sensors are defined by a three-dimensional grid of pressure evaluations evenly distributed around the spherical scatterer over a volume of $1 \mathrm{~m}^{3}$. Therefore, the error sensors, which define the control region, are external to the control sources and this control geometry is defined as an exterior control problem. For the active control and active cloaking control scenarios, the primary sound field is a plane wave approaching the sphere from an angle of $45^{\circ}$, as shown in Fig. 2a. In the case of sound field reproduction, the system attempts to reproduce the free field primary sound field, which is the plane wave approaching from $45^{\circ}$.

The optimal source strengths for the three control problems have been calculated according to Eqs. (5) (10) and (18) respectively and the regularisation, or control effort weighting parameter, $\beta$, has been set at each frequency in proportion to the eigenvalue spread of the matrix, $\mathbf{Z}$, which defines the response between the control sources and the error sensors. This regularisation has been used to ensure that the inverse problem is well conditioned. Fig. 3a shows the resulting attenuation in the respective cost functions given by Eqs. (2), (8) and (13) as a function of the non-dimensional wavenumber, $k a$, for the active control, sound field reproduction and active acoustic cloaking strategies. Whilst Fig. 3b shows the corresponding array effort given by the sum of squared control source strengths in each case.

From the results in Fig. 3 it can be seen that the active noise control and sound field reproduction strategies exhibit very similar performance over this frequency range. Both of these strategies are, as expected, most effective at relatively low frequencies where the acoustic wavelength is large, but also require the highest 
levels of control effort in this frequency range. From Eq. (6) it can be seen that the minimum of the cost function for the active noise control system is dependent on the total pressure field given by Eq. (19), whereas from Eq. (11) it can be seen that the sound field reproduction system is only dependent on the target sound field, which is the free field primary pressure, $\mathbf{p}_{p_{F}}$, in this case. The performance of these two systems is relatively similar, however, because the total pressure field is dominated by the free field incident pressure and so the dependence of the active noise control system performance on the scattered pressure contribution is relatively low. From the thick line in Fig. 3a it can be seen that the active acoustic cloaking strategy achieves reductions in the scattered pressure field in excess of $30 \mathrm{~dB}$ over the bandwidth considered and requires a much lower control effort than the other two sound field control strategies. These results indicate that the sphere has been effectively cloaked with a relatively low power requirement. The factors that limit this high level of control performance will be considered below, however, it is first interesting to observe the controlled sound fields for the three control strategies.

Fig. 4 shows the controlled sound field at $k a=3.7$, or $1 \mathrm{kHz}$ for the three control strategies. From Fig. 4a it can be seen that the active noise control system is only able to achieve effective reductions in the sound field in front of the sphere, in the direction of the incident primary plane wave. As a result, the total control at this frequency is relatively limited, as shown in Fig. 3 . A similar result can be seen in Fig. 4b, which shows the sound field produced by the sound field reproduction system at the same frequency. From this plot it can be seen that the system is able to approximately produce the required plane wave in front of the sphere, but is not able to accurately generate a plane wave to the rear of the sphere. In both the active noise control and sound field reproduction control scenarios the system is only effective in front of the sphere because the sound field is less influenced by the scatterer in this region. By contrast, Fig. 4c shows the sound field after active acoustic cloaking. From this plot it can be seen that the resulting sound field is a plane wave, and is indistinguishable from the free field plane wave shown in Fig. 2a. This confirms that system is able to achieve effective control of the scattered sound field, and effectively cloak the scattering sphere.

From the results presented in Fig. 3 it is clear that significant levels of attenuation in the scattered acoustic field are achievable over a relatively wide bandwidth, however, it is important to investigate how the control source density and geometrical distribution affect the control performance. Therefore, Fig. 5 shows the control performance at $k a=0.37$ as the number of control sources evenly distributed around the rigid sphere at a distance of $a / 20$ is increased from 1 to 180 . From this plot it can be seen that the performance of the active noise control and sound field reproduction systems initially rapidly increases as 


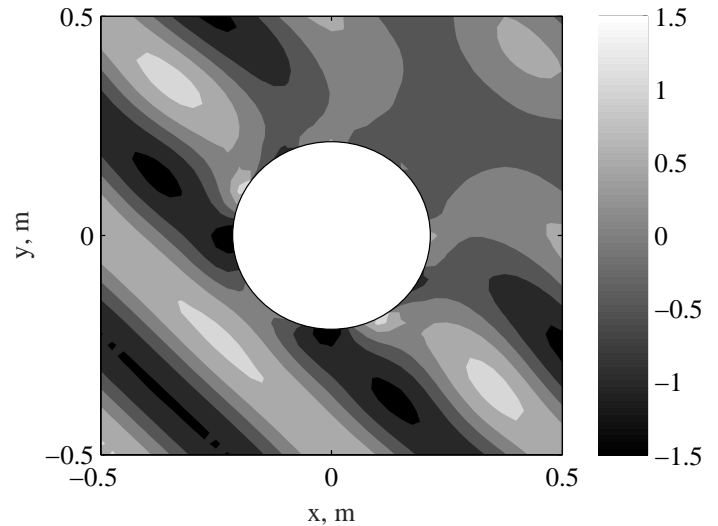

(a)

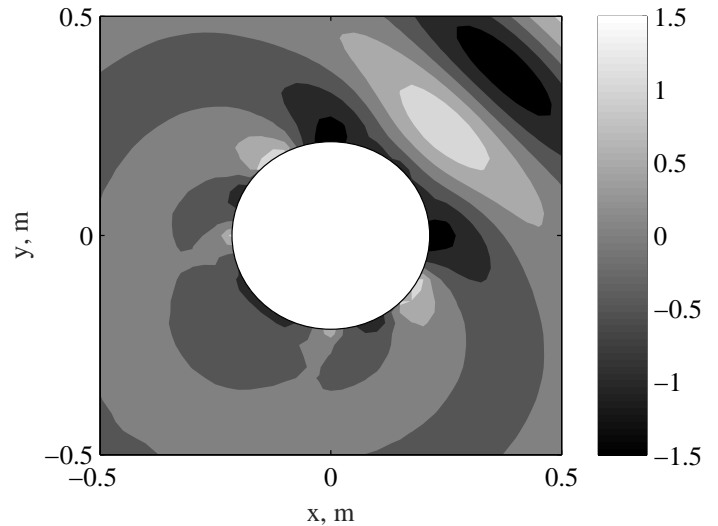

(b)

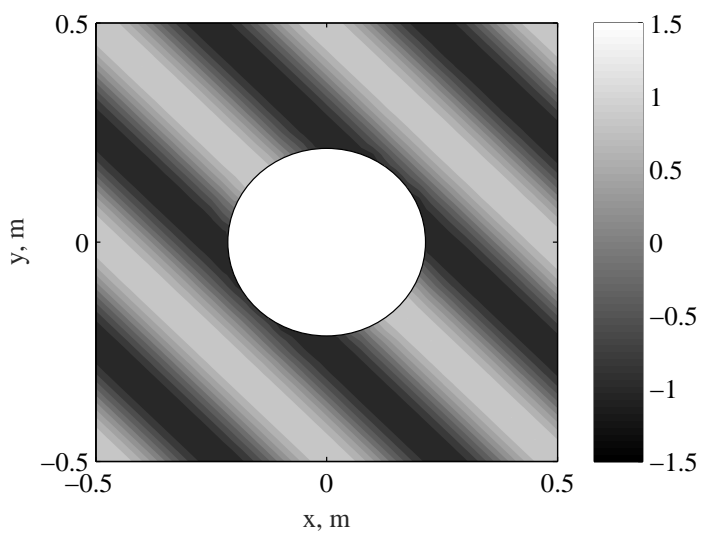

(c)

Figure 4: The controlled sound field at $k a=3.7$ or $1 \mathrm{kHz}$ for (a) active noise control (b) sound field reproduction and (c) active cloaking. 


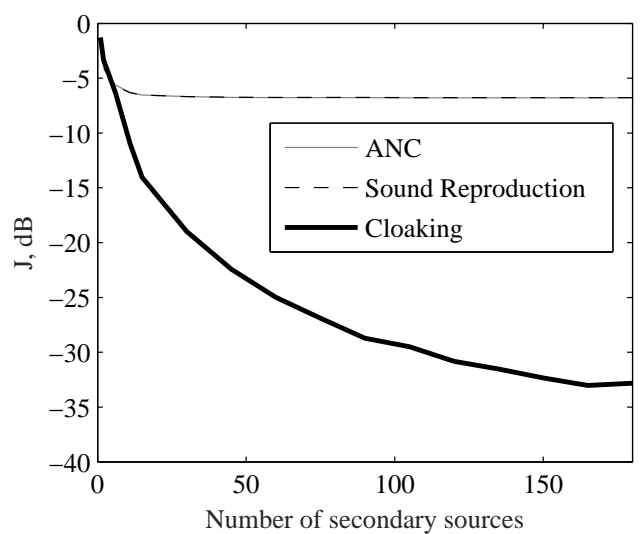

Figure 5: The attenuation in the cost functions for the Active Noise Control, Sound Field Reproduction and Active Acoustic Cloaking control schemes as the number of control sources uniformly distributed around the sphere is varied at $k a=0.37$.

the number of sources is increased from 1 to 15 , but then remains at around $6 \mathrm{~dB}$ as the number of control sources is increased further. This suggests that, beyond the initial increase in performance, these two control strategies are not significantly limited by the density of the control source distribution. Conversely, for the active acoustic cloaking system it can be seen that the performance continues to increase as the number of control sources is increased up to 180, at which point the cost function is reduced by more than $30 \mathrm{~dB}$. However, even with 15 control sources the scattered sound field cost function is reduced by around $15 \mathrm{~dB}$, suggesting that fewer control sources could be used in a practical system whilst obtaining significant levels of performance.

\section{B The Interior Control Problem}

In the previous section it has been shown that active cloaking is capable of achieving high levels of control performance when the control sources are located in close proximity to the scattering object. In contrast, the active noise control and sound field reproduction strategies achieve a relatively limited level of control for this exterior control problem. In many applications, sound field reproduction and active noise control have been applied to the interior control problem, which fulfils the Kirchoff-Helmholtz integral equation [1, 24, 25]. Therefore, in this section the case when the control sources are located at a distance of $2.5 a$ from the surface of the rigid sphere whilst the error microphone positions remain unchanged will be investigated; in this case the volume being controlled is bounded by the control source array and so this represents an interior control problem. 



Figure 6: The attenuation in the cost functions (a) and the corresponding control efforts (b) for the active noise control, sound field reproduction and active acoustic cloaking control schemes for a single layer array of 180 control sources uniformly distributed over a sphere at distance of $2.5 a$ from the scattering sphere controlling the interior sound field.

Fig. 6 shows the resulting levels of attenuation in the respective cost functions and the corresponding control efforts as a function of the non-dimensional wavenumber, $k a$, for the active control, sound field reproduction and active acoustic cloaking strategies. In this case it can be seen that the active noise control and sound field reproduction systems achieve much higher levels of attenuation than for the exterior control problem, whereas the active cloaking performance is limited to around $4 \mathrm{~dB}$ over the majority of the presented bandwidth. The increase in performance for the active noise control and sound field reproduction strategies can be related to the Kirchoff-Helmholtz integral equation, which states that the sound field inside of a volume can be perfectly reproduced by a continuous array of monopole and dipole sources on the boundary of that volume [26]. Although this condition is only approximately fulfilled here due to the finite number of monopole control sources, it explains why the performance for active noise control and sound field reproduction is significantly higher than for the exterior control problem in the previous section, which does not obey the assumptions of the Kirchoff-Helmholtz integral equation because the control sources do not enclose the control volume. For the active cloaking problem, because the primary sound field being controlled is generated by the scattering from the rigid sphere, in the case of the interior control geometry the primary source is inside of the control volume and, therefore, violates the assumptions of the Kirchoff-Helmholtz integral equation. Conversely, when considering the exterior active cloaking problem, the primary sound field generated by the scatterer is outside of the control volume, which has its surface boundaries defined at the control source array and at infinity, as shown in [1, p. 284]. 


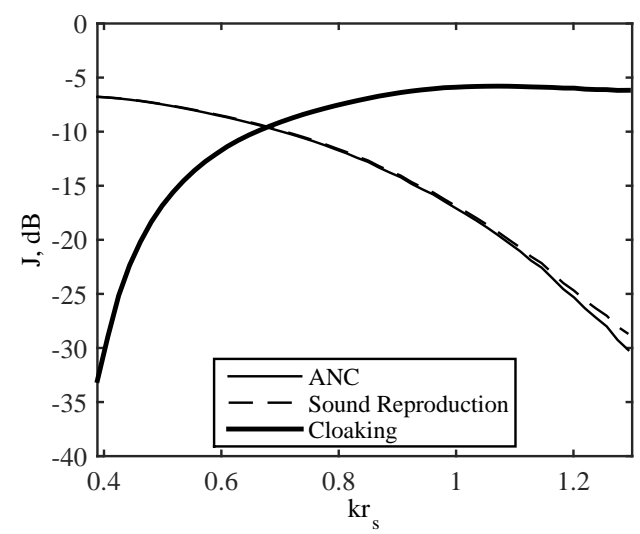

Figure 7: The attenuation in the cost functions for the active noise control, sound field reproduction and active acoustic cloaking control schemes as the distance between the array of control sources and the scattering sphere is increased at $k a=0.37$.

\section{The Transition Between Interior and Exterior Control Problems}

Further insight into the behaviour of the three control strategies can be gained by not only considering the two discrete cases of interior and exterior control as discussed above, but by investigating how the performance varies in each case as the distance between the control sources and the scatter is increased. Therefore, Fig. 7 shows the change in the cost functions for the three control systems employing 180 control sources as the radius of the array of control sources, $r_{s}$, is increased for $k a=0.37$; that is, as the distance between the control sources and the scattering sphere is increased.

From this plot it can be seen that when the control source array is close to the scatterer, as in the previous simulations, the performance of the active noise control and sound field reproduction systems is limited, while the active cloaking system achieves a high level of performance. However, as the distance between the rigid sphere and the control source array is increased, the performance of the active noise control and sound field reproduction systems significantly increases, while the cloaking performance reduces. This is consistent with the results presented in the previous sections, however, the results presented in Fig. 7 clearly demonstrate that in all three control strategies the control performance is directly affected by the distance between the control source array and the effective primary source. It is interesting to note that the performance of the three control strategies are comparable at around $k r_{s}=0.68$, which corresponds to a distance of a tenth of the acoustic wavelength. 


\section{A Non-Trivial Scattering Problem}

The results in the previous section have highlighted the physical limitations on the three different sound field control strategies and, in particular, have provided insight into how the limitations of active acoustic cloaking differ from those of active noise control and sound field reproduction. However, it is important to confirm how the above results for the simple spherical scattering object translate to a more complicated scatterer. Therefore, simulations have been conducted to investigate the interior and exterior control problems when the scattering object is a rigid cuboid with dimensions of $0.14 \mathrm{~m} \times 0.14 \mathrm{~m} \times 0.6 \mathrm{~m}$, as shown in Fig. 8. This problem has been modelled using the finite element method, as described in Section III and, once again, the primary sound field has been generated by a plane wave. The resulting sound field has been evaluated over a volume of $1 \mathrm{~m}^{3}$ using a three-dimensional grid of pressure evaluation points; a single horizontal plane of this full three-dimensional grid of measurement points is shown by the dots in Fig. 8. As in the previous sections, a minimum of 10 observation points per wavelength at the maximum frequency of interest are used to avoid limitations due to observability and consequently a total of 68921 pressure evaluations have been used to define the error sensor pressures.

The performance of the three sound field control strategies has first been calculated for the exterior control problem, where the control sources are located in close proximity to the cuboid scatterer as shown in Fig. 8. Fig. 9 shows the resulting levels of attenuation in the respective cost functions and the corresponding control efforts. From these results it can be seen that, as in the case of the simple spherical scatterer, high levels of control are achieved with a low control effort in the case of acoustic cloaking, whilst the performance of the active noise control and sound field reproduction systems is limited to low frequencies. In comparison to the results for the spherical scatterer it is clear that the cloaking performance is limited by the increased complexity of the scattering object, however, more than $12 \mathrm{~dB}$ of control is still achieved up to $k a=3$, which corresponds to $1 \mathrm{kHz}$.

Finally, the performance of the three sound field control strategies has been calculated for the interior control problem, where the control sources are located at a distance of $0.5 \mathrm{~m}$ from the surface of scattering cuboid, which is outside of the pressure evaluation grid. This corresponds to the interior control problem considered in Section B and the results for the scattering cuboid are shown in Fig. 10. From these results it can be seen that the same general behaviour occurs for the cuboid scatter as previously observed for the simple spherical scatterer. That is, in the case of the interior control problem, signifiant levels of performance are achieved for the active noise control and sound field reproduction systems, whilst the cloaking performance 


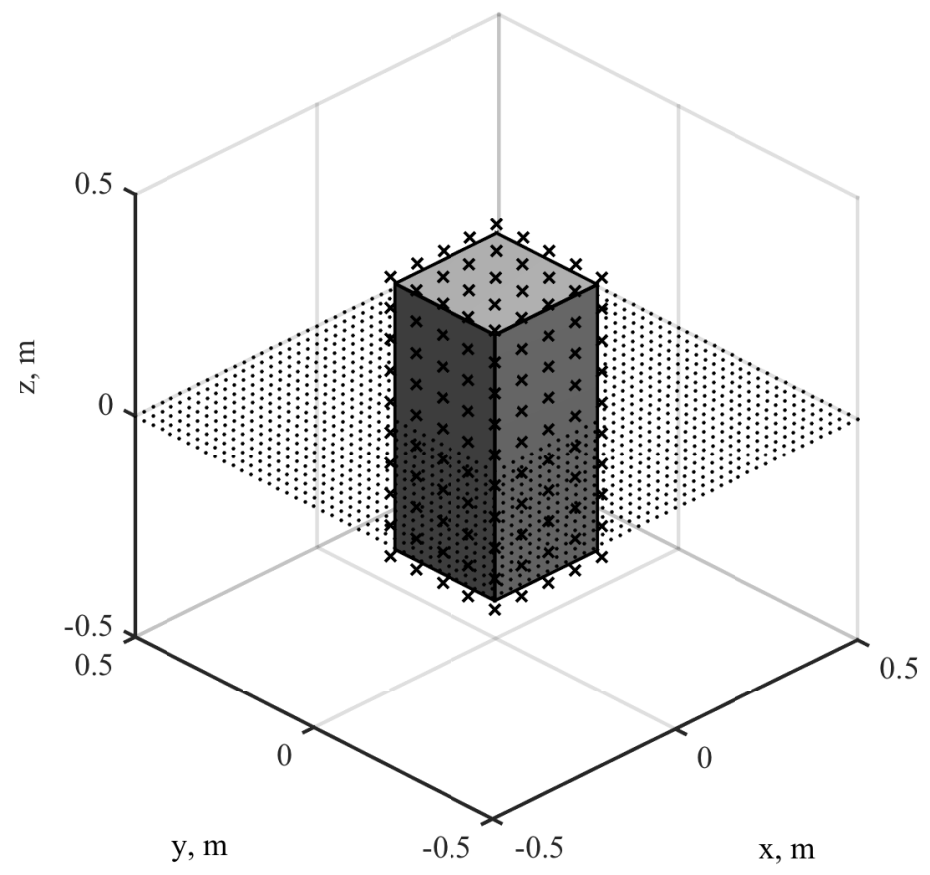

Figure 8: The geometry of the cuboid scatterer, the control sources for the exterior control geometry (crosses), and a single plane of the full three-dimensional grid of error sensors (dots).



(a)

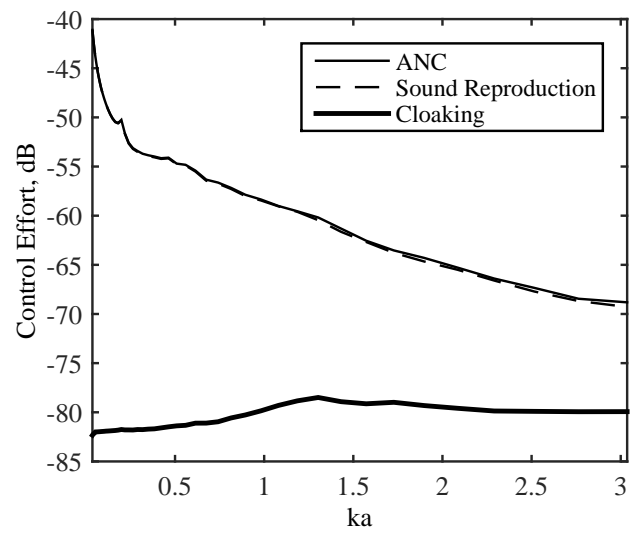

(b)

Figure 9: The attenuation in the cost functions (a) and the corresponding control efforts (b) for the active noise control, sound field reproduction and active acoustic cloaking control schemes for a single layer array of 180 control sources uniformly distributed over the scattering cuboid controlling the exterior sound field. 




(a)

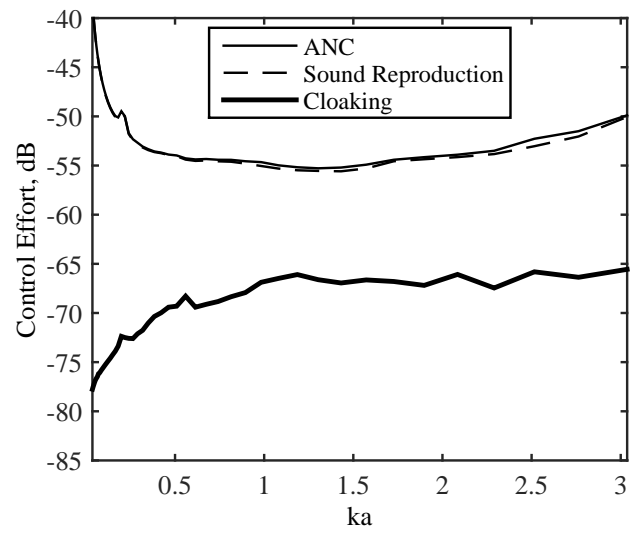

(b)

Figure 10: The attenuation in the cost functions (a) and the corresponding control efforts (b) for the active noise control, sound field reproduction and active acoustic cloaking control schemes for a single layer array of 180 control sources uniformly distributed over the scattering cuboid controlling the interior sound field.

is limited.

\section{Conclusions}

This paper has explored the physical limitations of active noise control, sound field reproduction and active acoustic cloaking in the context of controlling scattered sound fields. In order to first highlight the relationship between the three sound field control strategies, the three optimisation problems have been presented within a consistent framework in Section II. This has facilitated a direct comparison between the three strategies, which has not previously been presented in the literature. In particular, this comparison has highlighted that the optimal solution for active acoustic cloaking is the linear combination of the optimal solutions for active noise control and sound field reproduction. Although this suggests that active acoustic cloaking requires the cancellation of the primary disturbance and the reproduction of the sound field that would occur in the absence of the scatterer, it has been shown that, as expected, active acoustic cloaking only attempts to control the scattered component of the sound field.

In order to support the comparisons made between the three control strategy formulations, and investigate the physical limitations of the three strategies, a three-dimensional model of the acoustic scattering from a rigid sphere has been implemented and is described in Section III. This model has been used to investigate the three sound field control strategies and the results are presented in Section IV. These results demonstrate that 
when the control sources are located in close proximity to the scattering object, and are thus used to control the exterior sound field, a high level of acoustic cloaking is achieved over a wide bandwidth. Conversely, the performance of the active noise control and sound field reproduction systems is limited in this configuration, as it does not obey the assumptions of the Kirchoff-Helmholtz integral equation. When the control sources are located at a distance from the scattering sphere and they are used to control the interior sound field, which obeys the assumptions of the Kirchoff-Helmholtz integral equation, the active noise control and sound field reproduction systems achieve significant control performance. However, the active acoustic cloaking strategy achieves limited performance in this case, because the primary source generated by the scatterer is inside of the control volume and this violates certain assumptions of the Kirchoff-Helmholtz equation.

To provide further insight into the behaviour of the three control strategies, in addition to the two discrete control source array configurations, simulations have been conducted to investigate how the performance of the control strategies is influenced by the distance between the control sources and the scatterer. These results demonstrate that for the active acoustic cloaking system it is necessary for the control sources to be located in close proximity to the scatterer. That is, as the control sources are moved away from the scatterer the cloaking capability reduces. Conversely, for the active noise control and sound field reproduction systems, as the distance between the array of control sources and the scatterer is increased, the performance of both systems increases. In all three control scenarios, the trade-off between the performance and the location of the control source array can be simply related to the distance between the array and the dominant source that it is attempting to control or reproduce.

In addition to the effect of the distance between the control source array and scattering object, simulations have been conducted to investigate the influence of the number of control sources on the performance of the three control strategies for the exterior control problem. For the active noise control and sound field reproduction systems it has been shown that there is initially a significant increase in the performance as the number of control sources is increased to around 15 for the considered system, but the performance then shows only small improvements as the number of control sources is increased further. Conversely, for the active acoustic cloaking system the performance continues to increase as the number of control sources evenly distributed around the scatterer is increased.

Finally, in order to demonstrate the wider applicability of the conclusions of this work, a second sound field control problem has been considered in which the simple spherical scatterer has been replaced with a cuboid scatterer. It has been shown that the same performance limitations occur in this case for the three 
sound field control strategies. In particular, although the level of control achieved by the acoustic cloaking strategy is lower than for the spherical scatterer, significant levels of performance are still achieved over a wide bandwidth when the control sources are located in close proximity to the scatterer and used to control the exterior sound field.

Although the results presented in this paper are based on simulations of two acoustic scattering problems, this is probably the most effective means of investigating the physical limitations on the three sound field control problems within a consistent framework. The simulation based environment has allowed a large number of control source configurations to be investigated and, therefore, facilitated an investigation of how the number and distribution of control sources influences the control of acoustic scattering. In particular, this study has extended the work presented in $[3,17]$ by presenting a thorough investigation into the effects of control source geometry in active acoustic cloaking. This provides a basis for future experimental work, which should also consider the practical difficulties in evaluating the scattered sound field.

\section{References}

[1] P. Nelson and S. J. Elliott, Active Control of Sound, 436 (Academic Press, London) (1992).

[2] Y.-H. Kim and J.-W. Choi, "Sound field reproduction", in Sound Visualization and Manipulation, 283370 (John Wiley \& sons, Singapore) (2013).

[3] E. Friot and C. Bordier, "Real-time active suppression of scattered acoustic radiation", Journal of sound and vibration $\mathbf{2 7 8}, 563-580$ (2004).

[4] S. J. Elliott, I. M. Stothers, P. Nelson, M. A. McDonald, D. C. Quinn, and T. J. Saunders, "The active control of engine noise inside cars", in Proceedings of INTER-NOISE 88, edited by M. Bockhoff, volume 2, 987-990 (Poughkeepsie, New York) (1988).

[5] T. J. Sutton, S. J. Elliott, M. A. McDonald, and T. J. Saunders, "Active control of road noise inside vehicles", Journal of Noise Control Engineering 42, 137-146 (1994).

[6] S.-H. Oh, H. suk Kim, and Y. Park, "Active control of road booming noise in automotive interiors", Journal of the Acoustical Society of America 111, 180-188 (2002).

[7] J. Cheer and S. J. Elliott, "Multichannel control systems for the attenuation of interior road noise in vehicles", Mechanical Systems and Signal Processing 60-61, 753 - 769 (2015). 
[8] O. Kirkeby and P. A. Nelson, "Reproduction of plane wave sound fields", The Journal of the Acoustical Society of America 94, 2992-3000 (1993).

[9] S. J. Elliott, J. Cheer, H. Murfet, and K. R. Holland, "Minimally radiating sources for personal audio", The Journal of the Acoustical Society of America 128, 1721-1728 (2010).

[10] J. Cheer, S. J. Elliott, and M. F. S. Gálvez, "Design and implementation of a car cabin personal audio system", Journal of the Audio Engineering Society 61, 412-424 (2013).

[11] P. Coleman, P. J. Jackson, M. Olik, and J. A. Pedersen, "Personal audio with a planar bright zone", The Journal of the Acoustical Society of America 136, 1725-1735 (2014).

[12] M. A. Poletti and F. M. Fazi, "An approach to generating two zones of silence with application to personal sound systems", Journal of the Acoustical Society of America 137, 598-605 (2015).

[13] A. J. Berkhout, D. de Vries, and P. Vogel, "Acoustic control by wave field synthesis", The Journal of the Acoustical Society of America 93, 2764-2778 (1993).

[14] R. Rabenstein and S. Spors, "Sound field reproduction", in Springer Handbook of Speech Processing, 1095-1114 (Springer-Verlag Berlin, Heidelberg) (2008).

[15] C. Scandrett, "Scattering and active acoustic control from a submerged spherical shell", Journal of the Acoustical Society of America 111, 893-907 (2002).

[16] Y. Bobrovnitskii, "A new impedance-based approach to analysis and control of sound scattering", Journal of Sound and Vibration 297, $743-760$ (2006).

[17] E. Friot, R. Guillermin, and M. Winninger, "Active control of scattered acoustic radiation: a real-time implementation for a three-dimensional object", Acta acustica united with acustica 92, 278-288 (2006).

[18] N. Han, X. Qiu, and S. Feng, "Active control of three-dimension impulsive scattered radiation based on a prediction method", Mechanical Systems and Signal Processing 30, 267-273 (2012).

[19] Y. I. Bobrovnitskii, "A new solution to the problem of an acoustically transparent body", Acoustical Physics 50, 647-650 (2004).

[20] Y. I. Bobrovnitskii, "Impedance acoustic cloaking", New Journal of Physics 12, 043049 (2010). 
[21] M. Zampolli, A. Tesei, F. B. Jensen, N. Malm, and J. B. Blottman, "A computationally efficient finite element model with perfectly matched layers applied to scattering from axially symmetric objects", Journal of the Acoustical Society of America 122, 1472-1485 (2007).

[22] B. Rafaely, "Plane-wave decomposition of the sound field on a sphere by spherical convolution", Journal of the Acoustical Society of America 116, 2149-2157 (2004).

[23] L. Beranek and T. Mellow, Acoustics: sound fields and transducers, 489-503 (Academic Press, London) (2012).

[24] S. Ise, "A principle of sound field control based on the Kirchhoff-Helmholtz integral equation and the theory of inverse systems", Acta Acustica united with Acustica 85, 78-87 (1999)

[25] J. Ahrens and S. Spors, "An Analytical Approach to Sound Field Reproduction Using Circular and Spherical Loudspeaker Distributions", Acta Acustica united with Acustica 94, 988-999 (2008)

[26] A.J. Berkout, D. de Vries, and P. Vogel "Acoustic control by wave field synthesis", Journal of the Acoustical Society of America 93, 2764-2778 (1993). 